the MHC class II-deficient animals had a higher worm burden than control mice.

Similarly, T cell-deficient mice (athymic mice or $\mathrm{Tcrb}^{-/-}$mice) showed a significant expansion of the ISC pool and a decrease in enterocyte progenitors, suggesting again that ISC differentiation is diminished in the absence of ISC-T cell interactions. Finally, specific ablation of $\mathrm{T}_{\text {reg }}$ cells in mice resulted in a reduction in the ISC pool and an accumulation of more differentiated IECs (including enterocyte progenitors, tuft cells, goblet cells and the relative proportion of ISC-II and ISC-III subsets). A concomitant increase in $\mathrm{T}_{\mathrm{H}}$ cells near intestinal crypts of $\mathrm{T}_{\text {reg }}$ cell-deficient mice may contribute to the shift towards ISC differentiation. Together this suggests that signals from $\mathrm{T}$ cell populations balance ISC renewal and differentiation to support gut homeostasis in health and disease.

Lucy Bird

ORIGINAL ARTICLE Biton, $M$. et al. Thelper cel cytokines modulate intestinal stem cell renewal and differentiation. Cell https://doi.org/10.1016/j. cell.2018.10.008 (2018)

spiky + MPL-stimulated BMDMs completely blocked the growth of subcutaneous mouse tumours, whereas BMDM stimulation with MPL alone or plus rough particles or nanorods was much less effective. Similarly, mice immunized with influenza vaccine together with spiky particles plus MPL had higher levels of antibody and IFN $\gamma$ production compared with the use of clinically approved adjuvants such as alum and MF59. The mice that received the spiky + MPL adjuvant were the only ones to be fully protected from challenge with a lethal dose of influenza virus.

These results show the importance of physical cues as a second 'sense' by which innate immune cells activate the inflammasome. That a biological cue such as MPL is also required to prime the inflammasome should limit responses to non-biological particles.

Kirsty Minton

ORIGINAL ARTICLE Wang, J. et al. Physical activation of innate immunity by spiky particles. Nat. Nanotech. 13, 1078-1086 (2018)

\title{
CAR antigens beyond recognition
}

In clinical trials, $70-94 \%$ of patients with paediatric relapsed or refractory $B$ cell acute lymphoblastic leukaemia (B-ALL) show complete remission in response to anti-CD19 chimeric antigen receptor (CAR) T cell therapy (CTL019). Approximately $35 \%$ of responding patients eventually relapse. Two studies, both published in Nature Medicine, have now reported distinct mechanisms of resistance to CAR T cell therapy, as a result of loss of CD19 surface expression.

Orlando et al. analysed B-ALL samples at time of relapse from 17 patients, 12 of which had loss of surface CD19 expression.

RNA sequencing and DNA sequencing of the CD19-negative samples identified mutations in CD19 in the region encoding the extracellular and transmembrane domains (exons 2-5). Mutations in this region are predicted to produce a truncated protein without membrane anchorage. There were no relapse-associated mutations in other $B$ cell genes, thus relapse was specifically associated with CD19 mutations. The allelic frequencies of these mutations were proportionate to the percentage of CD19-negative cells in the B-ALL specimens, suggesting that nearly all tumour cells in the respective samples had a CD19 loss-of-function mutation. Furthermore, B cell populations in relapse patients were almost entirely CD19-negative, and loss of heterozygosity occurred in most of those patients. Other mechanisms of CD19 loss, such as alternative splicing or lineage switching, were excluded.

Ruella, Xu, Barrett et al. report the mechanism for relapse of a B-ALL patient, who, in frank relapse at around 9 months after CAR T cell therapy, had bone marrow infiltration of CD19-negative leukaemic cells as well as circulating CAR-transduced B cell leukaemia cells (CARB cells), and eventually died. Gene rearrangement analysis showed that CARB cells were clonally related to the original leukaemia, and thus the authors investigated whether relapse was due to lentiviral transduction occurring in vivo or during the CTL019 manufacturing process. The latter was confirmed to be the case. As such, the number of vector integration sites fell from 2,924 sites in the CTL019 infusion product to 2 integration sites comprising $97 \%$ of all sites sampled at time of relapse, indicating that transduced leukaemic blasts clonally expanded, leading to leukaemia relapse. Furthermore, this patient relapsed with CD19-negative leukaemia. Relapsed B-ALL blasts expressed CAR19 and CD19, which were colocalized on the cell surface, as detected by confocal microscopy. However, CD19 could not be detected by antibodies targeting its extracellular domain. This was due to CAR19 masking the CD19 epitope through binding to CD19 on the surface in cis, which mediated CD19-specific resistance of this clone to CTL019. Similarly, in vitro analysis showed that transduction of a B-ALL cell line with a CD22 CAR confers specific resistance to CD22 CAR and not to CD19 CAR. However, it remains unclear how unintended leukaemic cell transduction can be avoided during the CTL019 manufacturing process. Nonetheless, retrospective analysis of relapsed patients showed that the frequency of CD19 epitope masking in patients was very low, and none of the other relapsed patients had CAR19 expression in leukaemic cells.

Patients with relapsed CD19-negative leukaemia, in which CD19 surface expression is lost, might benefit from CAR T cell therapy targeting alternative antigens. However, the improvement of manufacturing techniques is required to minimize the risk of introducing CAR-transduced leukaemic cells that could confer specific resistance to therapy.

Ulrike Harjes, Nature Reviews Cancer This article is modified from the original in Nat. Rev. Cancer (https://doi.org/10.1038/s41568-018-0075-7).

ORIGINAL ARTICLE Orlando, E. J. et al. Genetic mechanisms of target antigen loss in CAR19 therapy of acute lymphoblastic leukemia. Nat. Med. 24, 1504-1506 (2018) | Ruella, M., Xu, J., Barrett, D. M. et al. Induction of resistance to chimeric antigen receptor T cell therapy by transduction of a single leukemic B cell. Nat. Med. 24, 1499-1503 (2018) 